\title{
25 Research Soure \\ Identification on the potential mechanism of Radix pueraria on colon cancer based on network pharmacology
}

Yi Li

Department of Clinical Laboratory, the First Affiliated Hospital of Zhengzhou University, Henan, China.

Chunli Zhang

Department of General Surgery, the People's Hospital of Zhengzhou, Henan, China.

\section{Xiaohan Ma}

Department of Clinical Laboratory, the First Affiliated Hospital of Zhengzhou University, Henan, China.

\section{Liuqing Yang}

Fuwai CentralChina Cardiovascular Hospital, Henan, China.

Huijun Ren ( $\nabla$ huijunren_2021@126.com )

Department of Clinical Laboratory, the First Affiliated Hospital of Zhengzhou University, Henan, China.

\section{Research Article}

Keywords: network pharmacology, colon cancer, Radix Puerariae, traditional chinese medicine, molecular docking

Posted Date: November 8th, 2021

DOI: https://doi.org/10.21203/rs.3.rs-1054829/v1

License: (1) This work is licensed under a Creative Commons Attribution 4.0 International License. Read Full License 


\section{Abstract}

Radix Puerariae (RP), a dry root of the Pueraria lobata (Willd.) Ohwi, is used to treat a variety of diseases, including cancer. Several in vitro and in vivo studies have demonstrated the efficacy of RP in the treatment of colon cancer (CC). However, the biological mechanism of RP in the treatment of colon cancer remains unclear. In this study, the active component of RP and its potential molecular mechanism against CC were studied by network pharmacology and enrichment analysis. The methods adopted included screening of active ingredients of Chinese medicine, prediction of target genes of Chinese medicine and disease, construction of protein interaction network, and GO and KEGG Enrichment Analysis. Finally, the results of network pharmacology were further validated by molecular docking experiments and cell experiments. 8 active constituents and 14 potential protein targets were screened from RP, including EGFR, JAK2 and SRC. The biological mechanism of RP against CC was analyzed by studying the relationship between active components, targets, and enrichment pathway. This provides a basis for understanding the clinical application of RP in CC.

\section{Introduction}

Colon cancer (CC) is the second most common cause of cancer death and the third most common cancer in the world ${ }^{1}$. The decline in the incidence and mortality of colon cancer in developed countries may be associated with increased cancer screening in developed countries. However, the rapid rise in mortality rates in developing countries and the fact that more than half of the patients were diagnosed as latestage significantly increased the medical and economic burden in developing countries ${ }^{2}$. Despite progress in treatment and care in recent years, many treatments are still accompanied by severe adverse reactions and drug resistance, which can reduce the quality of life and increase patient suffering. Therefore, adjuvant therapy and alternative therapies with low toxicity are necessary to improve quality of life and prolong life time. Traditional Chinese Medicine (TCM), as a critical adjuvant to tumor therapy, can improve the effectiveness of treatment, reduce drug resistance, and prolong survival time ${ }^{3}$. For example, adjuvant treatment with Chinese herbal medicine can reduce the hepatotoxicity of colon cancer patients 4 . Furthermore, alkaloids can inhibit the growth of colon cancer cells through Wnt/ $\beta$-catenin signaling pathway ${ }^{5}$. Previous studies have shown that traditional Chinese medicine Angelica can increase the sensitivity of colon cancer cells to radiotherapy and chemotherapy. In addition, traditional Chinese medicine ginsenoside $\mathrm{Rh} 2$ can reduce the resistance of colon cancer cells to oxaliplatin ${ }^{6}$.

Radix Puerariae (RP) is a kind of Chinese medicine that was used for medicine in Jin Dynasty. RP is a kind of dried root of the Pueraria lobata (Willd.) Ohwi, which has the effects of reducing heat, stopping diarrhea, producing body fat, and benefiting ${ }^{7}$. Now RP is used in the treatment of many diseases such as angina, hypertension, diabetes, optic atrophy, and retinitis ${ }^{8}$. RP is also commonly used in the treatment of cancer. In vitro experiments confirmed the anticancer activity of most components of RP, such as puerariae radix isoflavones, inhibit the growth of breast cancer cells ${ }^{9}$; puerarin, for example, inhibits metastasis and invasion of liver cancer through PTEN/AKT signaling ${ }^{10}$. In modern medicine, RP is also 
often used for the treatment of colon cancer, but the active components and targets of RP treatment for colon cancer are not known.

Network pharmacology can show the complex relationship between drug and disease in the form of network. In the network, drug target-disease target-pathways network and protein-protein Interaction (PPI) network are commonly used to predict the efficacy of drug to disease. The degree and clustering coefficient are common parameters for evaluating the network. ADME system refers to the process of drug absorption, distribution, metabolism, and excretion in the body ${ }^{11-13}$. The ADME-based admetSAR method was proved to be a successful method. We selected the parameters of HIA, Caco-2 and HOB in the admetSAR database to optimize the active ingredient screening strategy.

In the 19th century, the receptor theory was proposed to consider the pairing of drug small molecules as ligands and protein macromolecules as receptors to form keys and locks ${ }^{14}$. With the establishment of energy matching and geometric complementary models for ligands and receptors and the development of computer technology, the idea of simulating the interaction between ligands and receptors is realized, namely molecular docking analysis. Molecular docking refers to the prediction of binding patterns of ligand small molecules and receptor proteins by continuously optimizing their conformations, positions, amino acid residues and other binding modes when both ligand small molecules and receptor proteins are three-dimensional structures and the minimum energy of ligand small molecules ${ }^{15}$. The method is to screen suitable drugs by scoring affinity.

In this study, the active ingredient of RP and its possible anti-cancer mechanism were studied using network pharmacology. Key targets and pathways were experimentally verified by molecular docking and in vitro validation. The study flow is shown in the figure below (Fig. 1).

\section{Results}

\section{Screening active ingredients of RP}

After combining the RP ingredients found in the following databases, there were 40 components, of which 15 components were retrieved from the TCPSP database, 12 components were retrieved from the ETCM database, 10 components were retrieved from the herb database and 3 components were retrieved from the literature (Supplementary Table S1). The retrieved ingredients were submitted to the admetSAR website (http://Immd.ecust.edu.cn/admetsar2) for further screening based on the results of human intestinal absorption (HIA), Caco-2, human oral bioavailability (HOB), and plasma protein binding (PPB) $\triangle$ Supplementary Table S2】. The results showed that there were 9 components with good absorption and distribution properties, and their chemical constituents are mainly isoflavone, coumarins, and alkaloids (Table 1). Although the Caco-2 and HOB of puerarin predicted by admetSAR were lower, it has an important biological role in previous studies, so it was temporarily retained for further study.

\section{Intersection of Related Targets}


SwissTargetPrediction is a network tool designed to predict the most likely protein targets in small molecules by reverse screening based on similarity principles. Submit the SMILES descriptions of puerarin and the screened active ingredients to the SwissTargetPrediction database and output the results with the similarity probability $>0$ to the target (Supplementary Table S3). By combining the targets of active ingredient and deleting duplicates, we obtained 226 targets related to RP. A total of 21,894 colon cancer-related targets were obtained from GeneCards and OMIM. Then we inputted RP and CC targets to Venny 2.1, and the results showed that there were 219 common gene targets between RP and CC (Supplementary Table S4) (Fig. 2).

\section{Analysis of Target PPI Network}

The STRING database is used to show the link between proteins participating in specific biological functions. We entered common targets into the "Multiple proteins" of STRING database, selected the highest confidence level and hided the disconnected nodes in the network to obtain the interaction between them. Then the results of TSV format were imported into Cytoscape, and 104 nodes and 218 edges were displayed (Fig. 3). By calculating and visualizing the targets degree values in PPI network, the genes with higher degree are SRC, LYN, JAK2, MAPK14, MAPK8, PTK2, PTK2B, EGFR, NFKB1, JAK1, PTPN6, SYK, FGR and ESR1.

\section{GO and KEGG Analysis}

We used Metascape for GO function and KEGG pathway analysis to further understand the mechanism of RP on CC, and set $\mathrm{P}$ Value $<0.01$ to be significant. $\mathrm{GO}$ function results includes three parts: biological process (BP), cell component (CC) and molecular function (MF). In BP GO terms, peptidyl-tyrosine phosphorylation, regulation of cell adhesion, response to drug et al. may be associated with tumor regulation. $\mathrm{CC}$ terms are mainly enriched in perinuclear region of cytoplasm, membrane raft, focal adhesion, etc. In MF GO terms, protein kinase activity, kinase binding, and transmembrane receptor protein tyrosine kinase activity may be associated with tumors $\bigotimes$ Fig. 4囚. In KEGG enrichment pathways, EGFR tyrosine kinase inhibitor resistance, PI3K-Akt signaling pathway, apoptosis, and NF-kappa B signaling pathway is involved in apoptosis and cancer regulation (Fig. 5). These pathways may be critical in the treatment of CC. The results of KEGG analysis were compared with PPI network, and 7 targets with higher degree were randomly selected for receptor proteins in molecular docking, including SRC, JAK2, MAPK14, EGFR, NFKB1, ESR1, and IL2.

\section{Compounds Target Network Analysis}

The first 20 pathways with the largest number of genes were chosen to construct a RP-componentstargets-pathways network with 183 nodes and 626 edges $\varangle$ Supplementary Table S5囚(Fig. 6). The red node refers to drug; The green node represents pathways; The blue nodes represent targets; The yellow nodes indicate components. The edges indicate their interactions. Each compound interacts with multiple targets in the graph, suggesting that the effect of RP on CC may be a synergistic effect of multiple targets. According to the degree of topological parameters of network, five high-level components were 
selected as ligand molecules in molecular docking. Of the five ingredients, three isoflavones component, namely formononetin, daidzein, and 3'-Methoxydaidzein; one alkaloid component, namely sitosterol; and one coumarin component, namely scoparone.

\section{Molecular Docking Analysis}

The PDB Entry number of the targets structure selected from the PDB database is EGFR (5UG9), JAK2(3UGC), MAPK14(2FST), NFKB1(1SVC), ESR1(3CBP), IL2(4NEJ), and SRC (1043). We conducted molecular docking between receptor proteins and ligand molecules through AutoDockTools 1.5.6. The result of AutoDockTools was output in the form of affinity score, which is the core parameter of AutoDockTools (Table 2). The lower the affinity score, the better the binding effect. PYMOL software visualized the docking complexes and binding residues of 3'- Methoxydaidzein and formononetin ligand molecule with MAPK14 receptor protein (Fig. 7).

\section{RP inhibits proliferation of colon cancer cells}

The anti-cancer effect of RP at different concentrations $(0,5,10,15$, and $20 \mu \mathrm{g} / \mathrm{ml})$ on SW480 cells for 24,48 and 72 hours was verified by CCK-8 experiment. The proliferation of SW480 cells treated with different concentrations of RP decreased in a dose- and time-dependent manner冈Fig. 8囚. The proliferation ability of SW480 cells decreased linearly under low concentration of RP, but did not change much under high concentration of RP. The IC50 values of 24,48 and $72 \mathrm{~h}$ after RP treatment were $14.9,9.8$, and 8.0 $\mu \mathrm{g} / \mathrm{ml}$ in SW480 cells, respectively.

\section{RP inhibits migration of colon cancer cells}

The effect of RP on SW480 cells migration was studied by scratch test (Fig. 9). As shown in the figure, at $12 \mathrm{~h}$, the cell scratches of the three groups were reduced by different treatment methods, and the reduction range of the control group was the largest; at $48 \mathrm{~h}$, the control group was further reduced by a large margin, but there was no significant change in the two groups after treatment with RP medium. The results showed that the effect of RP on SW480 cell migration was more significant over time than that of the control group. It suggested that RP could inhibit the invasion and migration activity of SW480 cells.

\section{Discussion}

Cancer is a common disease with complex pathogenesis, characterized by abnormal cell growth and uncontrolled division. With the emergence of problems such as side effects and drug resistance of anticancer drugs, it has become a hot spot to find natural anticancer drugs with lower side effects. As a natural product with abundant resources, traditional Chinese medicine has attracted more and more attention because of its unique advantages in reducing the side effects of anticancer drugs, prolonging the survival rate of patients, and improving life of patients ${ }^{16}$. Because of the complex composition of natural products, the development and use of traditional Chinese medicine also face new problems. 
Therefore, the screening method based on ADME characteristics has been widely recognized in the development and application of traditional Chinese medicine ${ }^{17}$.

$\mathrm{RP}$ is a kind of Chinese herb with anticancer, anti-inflammatory, anti-oxidant and cardiovascular protection. RP is mainly used in the treatment of cancer, endocrine disease, cardiovascular disease, and neurodegeneration. The results of RP component screening showed that the activity of isoflavones was the strongest, followed by alkaloid and coumarin. Previous studies on the anti-cancer effects of RP have focused primarily on the role of puerarin, with few studies on other active components of RP ${ }^{18-21}$. Studies on formononetin, daidzein and other RP components show that they can also inhibit growth and induce apoptosis in human colon cancer cells. Formononetin in vivo experiments show that it can inhibit the growth and proliferation of human colon cancer cells, reduce the invasiveness and vascular endothelial growth factor (VEGF) expression level in tumor tissues ${ }^{22}$. The genotoxic and cytotoxic effects of daidzein on human colon adenocarcinoma cells are dose-dependent ${ }^{23}$. These RP active ingredients were successfully screened by ADME parameters.

PPI network analysis of common targets for RP and colon cancer showed that 14 targets had a high degree of SRC, LYN, JAK2, MAPK14, MAPK8, PTK2, PTK2B, EGFR, NFKB1, JAK1, PTPN6, SYK, FGR and ESR1.In molecular docking experiments, EGFR and 3'- Methoxydaidzein showed high affinity, indicating that EGFR may play an important role in RP treatment of colon cancer.

Epidermal growth factor receptor (EGFR) is a cancer-promoting tumor marker that regulates the homeostasis and growth of epithelial tissues and cells. EGFR is also considered to be associated with the development of tumor resistance due to the occurrence of point mutations and amplification following the use of anticancer drugs ${ }^{24}$. EGFR is a member of receptor tyrosine kinase (RTK), a family of proteins that needs to form heterodimer to function. The up-regulation of EGFR activity is mediated by common truncated and mutated extracellular domains, and the up-regulated EGFR over-activates the downstream pro-cancer signaling pathways, including AKT-PI3K-mTOR and RAS-RAF-MEK-ERK MAPK pathways ${ }^{25}$. These pathways then activate a variety of biological effects that are beneficial to the proliferation of cancer cells, causing cancer to occur and develop. This evidence suggests that RP may play a role in the treatment of colon cancer by down-regulating EGFR protein expression.

In the GO functional analysis, we screened the first 20 terms out of the three parts BP, CC and MF based on $\mathrm{P}$ Value $<0.01$. In BP GO terms, peptidyl-tyrosine phosphorylation, response to drug, $\mathrm{CC}$ terms membrane raft, and In MF GO terms, protein kinase activity, kinase binding, and transmembrane receptor protein tyrosine kinase activity may be associated with tumors. Then we screened the first 20 pathways obtained from KEGG enrichment analysis according to the criteria of $P$ Value $<0.01$. The pathway with the most enriched genes is PI3K-Akt signaling pathway, with 22 targets enriched on this pathway, including EGFR, HSP90AB1, IL2, JAK2, NFKB1, MAPK14, SRC, etc. The phosphatidylinositol 3'-kinase (PI3K)-Akt is a signaling pathway that regulates the basic functions of cells, such as transcriptional translation and growth and proliferation, which can be activated by toxic damage or cell stimulation ${ }^{26,27}$. PI3K catalyzes the formation of phosphatidylinositol 3,4,5-triphosphate (PIP3) on the cell membrane, and PIP3 acts as a 
second messenger to activate Akt. Finally, Akt regulates the key processes of cell growth and development by phosphorylation of proteins involved in cell synthesis, metabolism, cell cycle and apoptosis. RP may play an anti-CC role by regulating proteins enriched in PI3K-Akt signaling pathway.

\section{Methods}

\section{Screening of active ingredient}

The components of RP were obtained from Traditional Chinese Medicine Systems Pharmacology Database and Analysis Platform ${ }^{28}$ (https://tcmspw.com/tcmsp.php), the Encyclopedia of Traditional Chinese Medicine ${ }^{29}$ (http://www.tcmip.cn/ETCM/index.php/Home/), Herb ${ }^{30}$ (http://herb.ac.cn/), and literature. Then attain the Canonical SMILES of these ingredients from NCBI PubChem ${ }^{31}$ (https://pubchem.ncbi.nIm.nih.gov). Finally, the SMILES descriptions were input into admetSAR ${ }^{32}$ (http://Immd.ecust.edu.cn/admetsar2/) to screen active components of RP according to HIA, Caco-2 and HOB. Ingredients that did not meet the screening requirements but were significantly bioactive in previous studies were retained.

\section{Acquisition of Gene Targets}

The screened active ingredients smiles were uploaded to the Swiss Target Prediction ${ }^{33}$ (http://www.swisstargetprediction.ch/) to predict the target, the species was set as Homo sapiens, and the result of probability $>0.1$ was selected. At the same time, colon cancer associated target genes were obtained from GeneCards ${ }^{34}$ (https://www.genecards.org/) and Online Mendelian Inheritance in Man (https://omim.org/) ${ }^{35}$, and the results of colon cancer-target genes from both databases were combined. Then we used Venny 2.1 (http://bioinfogp.cnb.csic.es/tools/venny/index.html) to obtain the gene target intersection of RP and colon cancer.

\section{Construction of Protein-protein Interaction (PPI) Network}

Gene targets overlapped by RP and CC were input into STRING database ${ }^{36}$ (https://string-db.org) to obtain PPI network. Parameter is set to Homo sapiens and the interactive score is set to highest credibility. Then the target intersection file was imported into the Cytoscape3.7.1 for visual analysis of PPI network.

\section{GO and KEGG Enrichment Analysis}

We submitted the intersection target genes to Metascape ${ }^{37}$ (http://metascape.org), selected "H. sapiens" as the input species, set $P$ Value $\leq 0.01$, and then conducted the Gene ontology (Go) terms and Kyoto Encyclopedia of Genes and Genomes (KEGG) pathway enrichment analysis. The first 20 higher-score GO enrichment or KEGG pathways were analyzed ${ }^{38}$.

\section{Construction of Interactive Network}


In order to understand the relationship between components, targets, and enrichment pathways, we use Cytoscape3.7.1 $1^{39}$ to conduct visual analysis and construct interactive network.

\section{Molecular Docking}

According to the interactive network results of RP components and targets, the molecular docking experiments were carried out at the nodes with larger degree. The 2D SDF structure files of components were obtained by NCBI PubChem, and the SDF files were input into Chem3D 20.0 software to minimize energy. The targets were entered into the UniProt ${ }^{40}$ (https://www.uniprot.org) database, filtered by reviewed and human organisms, and then the lower resolution, single chain and the X-ray pathway were preferred to selected according to the structure information. The selected crystal structures were downloaded from the Protein Data Bank database (http://www.rcsb.org/pdb) in pdb format ${ }^{41,42}$. Then the targets were de-liganded and de-watered by PYMOL 2.3.4 software, hydrogenated and charge balanced by AutoDockTools 1.5.6 software, and treated by Grid Option tool. Finally targets and components were converted into pdbqt format respectively. We used AutoDockTools to evaluate the affinity of components and target proteins. The docking complexes of ligand and receptor proteins and their binding residues were visualized by PYMOL software ${ }^{43,44}$.

\section{Cell Culture}

The human colon cancer cell SW480 used in this study were obtained from the cell library of the Chinese Academy of Sciences (Shanghai, China). The cells were preserved in Dulbecco modified Eagle medium (DMEM) containing 1\% penicillin/streptomycin (Corning) and 10\% fetal bovine serum (Gibco).

\section{CCK-8 assay}

The cell viability of CC cells was determined by Cell Counting Kit-8 (CCK-8) assay. SW480 cells were inoculated into 96 -well plates at a density of $4 \times 10 /{ }^{4} \mathrm{ml}$. After overnight culture, the cells were treated with $\mathrm{RP}$ at $0,5,10,15,20 \mu \mathrm{g} / \mathrm{ml}$ in DMEM medium for 24,48 , and 72 hours. The cells were then incubated with Cell Counting Kit- 8 at $37^{\circ} \mathrm{C}$ for 2.5 hours in a $5 \%$ carbon dioxide incubator, and their absorbance values at $450 \mathrm{~nm}$ were measured. The results were analyzed and plotted with Graphpad prism 8.0 software.

\section{Scratch test}

SW480 cells in exponential growth phase were inoculated in 6 -well plates $\left(5 \times 10^{5}\right.$ cells/well). When the cells reached a state of fusion into a monolayer, 3 uniform lines were drawn vertically along the plate with a pipette aspirator with a sterile diameter of $2 \mathrm{~mm}$. The cells were then incubated for 0,12 , and $48 \mathrm{~h}$ with drug-free medium (serum-free medium) and drug-containing medium (serum-free medium containing 5 , $10 \mu \mathrm{g} / \mathrm{ml}$ of RP). Each group was repeated three times. Photographs were taken with an inverted microscope (Leica, Germany) to observe the migration rate of cells in each group.

\section{Statistical Analysis}


All data are measured in three separate experiments, expressed as mean $\pm \mathrm{SD}$. The differences between the treatment and control groups were analyzed by Graph Pad Prism8.0(GraphPad Software Inc., USA) one-way analysis of variance (ANOVA). $P<0.05$, the difference was statistically significant.

\section{Declarations}

\section{Funding}

This work was supported by the National Natural Science Foundation of China (No.81401682), Programs for Science and Technology Development of Henan Province (172102310649), and the Key Scientific Research Program of Higher Education of Henan Province (19B310006).

\section{Data availability}

Supplementary files are provided for drug targets, ADME screening results, common targets for drugs and diseases, and RP- components - targets - pathways network results.

\section{Author contributions}

R.H.J. and L.Y. designed the project and performed cell experiment validation. Z.C.L. and M.X.H. collected and analyzed the data. Y.L.Q. made a graphic drawing and L.Y. wrote the article. In addition, all authors read and agreed to the paper.

\section{Competing interests}

The author states that there is no competitive interest.

\section{Additional information}

Correspondence and requests for materials should be addressed to R.H.J.

\section{References}

1. Chang, K.-F. et al. Antitumor Effects of N-Butylidenephthalide Encapsulated in Lipopolyplexs in Colorectal Cancer Cells. Molecules 25, E2394 (2020).

2. Uyemura, S. A., Stopper, H., Martin, F. L. \& Kannen, V. A Perspective Discussion on Rising Pesticide Levels and Colon Cancer Burden in Brazil. Front Public Health 5, 273 (2017).

3. Wang, Z. et al. An update on Chinese herbal medicines as adjuvant treatment of anticancer therapeutics. Biosci Trends 12, 220-239 (2018).

4. Lin, T.-H. et al. The use of Chinese herbal medicine as an adjuvant therapy to reduce incidence of chronic hepatitis in colon cancer patients: A Taiwanese population-based cohort study. J Ethnopharmacol 202, 225-233 (2017). 
5. Ren, H. et al. Alkaloids from nux vomica suppresses colon cancer cell growth through $\mathrm{Wnt} / \beta$-catenin signaling pathway. Phytother Res 33, 1570-1578 (2019).

6. Ma, J. et al. Reversal effect of ginsenoside Rh2 on oxaliplatin-resistant colon cancer cells and its mechanism. Exp Ther Med 18, 630-636 (2019).

7. Zhang, L. Pharmacokinetics and drug delivery systems for puerarin, a bioactive flavone from traditional Chinese medicine. Drug Deliv 26, 860-869 (2019).

8. Zhou, Y.-X., Zhang, H. \& Peng, C. Puerarin: a review of pharmacological effects. Phytother Res 28, 961-975 (2014).

9. Lin, Y.-J. et al. Puerariae radix isoflavones and their metabolites inhibit growth and induce apoptosis in breast cancer cells. Biochem Biophys Res Commun 378, 683-688 (2009).

10. Zhou, Y., Xue, R., Wang, J. \& Ren, H. Puerarin inhibits hepatocellular carcinoma invasion and metastasis through miR-21-mediated PTEN/AKT signaling to suppress the epithelial-mesenchymal transition. Braz J Med Biol Res 53, e8882 (2020).

11. Fan, J. \& de Lannoy, I. A. M. Pharmacokinetics. Biochem Pharmacol 87, 93-120 (2014).

12. Yang, Z., Kulkarni, K., Zhu, W. \& Hu, M. Bioavailability and pharmacokinetics of genistein: mechanistic studies on its ADME. Anticancer Agents Med Chem 12, 1264-1280 (2012).

13. Vugmeyster, Y., Harrold, J. \& Xu, X. Absorption, distribution, metabolism, and excretion (ADME) studies of biotherapeutics for autoimmune and inflammatory conditions. AAPS J 14, 714-727 (2012).

14. Comeau, S. R., Gatchell, D. W., Vajda, S. \& Camacho, C. J. ClusPro: an automated docking and discrimination method for the prediction of protein complexes. Bioinformatics 20, 45-50 (2004).

15. Nagamine, N. \& Sakakibara, Y. Statistical prediction of protein chemical interactions based on chemical structure and mass spectrometry data. Bioinformatics 23, 2004-2012 (2007).

16. Y, L. et al. Cellular senescence and cancer: Focusing on traditional Chinese medicine and natural products. Cell proliferation 53, (2020).

17. H, L., J, W., W, Z., Y, W. \& L, Y. Systems approaches and polypharmacology for drug discovery from herbal medicines: an example using licorice. Journal of ethnopharmacology 146, (2013).

18. Z, Y. \& W, L. Induction of apoptosis by puerarin in colon cancer HT-29 cells. Cancer letters 238, (2006).

19. X, L., W, Z., W, W., S, L. \& L, Y. Puerarin suppresses LPS-induced breast cancer cell migration, invasion and adhesion by blockage NF-kB and Erk pathway. Biomedicine \& pharmacotherapy = Biomedecine \& pharmacotherapie 92, (2017).

20. T, Y. et al. Synthesis, characterization, and formulation of poly-puerarin as a biodegradable and biosafe drug delivery platform for anti-cancer therapy. Biomaterials science 7, (2019).

21. Deng, X.-Q. et al. Colon-specific microspheres loaded with puerarin reduce tumorigenesis and metastasis in colitis-associated colorectal cancer. Int J Pharm 570, 118644 (2019). 
22. Kc, T. et al. Formononetin: A Review of Its Anticancer Potentials and Mechanisms. Frontiers in pharmacology 10, (2019).

23. Salama, A. A. A. \& Allam, R. M. Promising targets of chrysin and daidzein in colorectal cancer: Amphiregulin, CXCL1, and MMP-9. Eur J Pharmaco/ 892, 173763 (2021).

24. Sigismund, S., Avanzato, D. \& Lanzetti, L. Emerging functions of the EGFR in cancer. $\mathrm{Mol}$ Oncol 12, 3-20 (2018).

25. Wee, P. \& Wang, Z. Epidermal Growth Factor Receptor Cell Proliferation Signaling Pathways. Cancers (Basel) 9, E52 (2017).

26. The relation between PI3K/AKT signalling pathway and cancer - PubMed. https://pubmed.ncbi.nlm.nih.gov/30849534/.

27. Porta, C., Paglino, C. \& Mosca, A. Targeting PI3K/Akt/mTOR Signaling in Cancer. Front Onco/ 4, 64 (2014).

28. Ru, J. et al. TCMSP: a database of systems pharmacology for drug discovery from herbal medicines. J Cheminform 6, 13 (2014).

29. Xu, H.-Y. et al. ETCM: an encyclopaedia of traditional Chinese medicine. Nucleic Acids Res 47, D976D982 (2019).

30. Fang, S. et al. HERB: a high-throughput experiment- and reference-guided database of traditional Chinese medicine. Nucleic Acids Res 49, D1197-D1206 (2021).

31. PubChem Substance and Compound databases - PubMed. https://pubmed.ncbi.nlm.nih.gov/26400175/.

32. Yang, H. et al. admetSAR 2.0: web-service for prediction and optimization of chemical ADMET properties. Bioinformatics 35, 1067-1069 (2019).

33. Gfeller, D. et al. SwissTargetPrediction: a web server for target prediction of bioactive small molecules. Nucleic Acids Res 42, W32-38 (2014).

34. Safran, M. et al. GeneCards Version 3: the human gene integrator. Database (Oxford) 2010, baq020 (2010).

35. Amberger, J. S., Bocchini, C. A., Schiettecatte, F., Scott, A. F. \& Hamosh, A. OMIM.org: Online Mendelian Inheritance in Man (OMIM®), an online catalog of human genes and genetic disorders. Nucleic Acids Res 43, D789-798 (2015).

36. von Mering, C. et al. STRING: a database of predicted functional associations between proteins. Nucleic Acids Res 31, 258-261 (2003).

37. Zhou, Y. et al. Metascape provides a biologist-oriented resource for the analysis of systems-level datasets. Nat Commun 10, 1523 (2019).

38. Chen, L. et al. Prediction and analysis of essential genes using the enrichments of gene ontology and KEGG pathways. PLoS One 12, e0184129 (2017).

39. Cytoscape StringApp: Network Analysis and Visualization of Proteomics Data - PubMed. https://pubmed.ncbi.nlm.nih.gov/30450911/. 
40. UniProt Consortium. UniProt: a worldwide hub of protein knowledge. Nucleic Acids Res 47, D506D515 (2019).

41. Berman, H. M. et al. The Protein Data Bank. Nucleic Acids Res 28, 235-242 (2000).

42. Burley, S. K. et al. Protein Data Bank (PDB): The Single Global Macromolecular Structure Archive. Methods Mol Biol 1607, 627-641 (2017).

43. Bioinformatics, Molecular Docking and Experiments In Vitro Analyze the Prognostic Value of CXC Chemokines in Breast Cancer - PubMed. https://pubmed.ncbi.nlm.nih.gov/34123826/.

44. Morris, G. M. et al. AutoDock4 and AutoDockTools4: Automated docking with selective receptor flexibility. J Comput Chem 30, 2785-2791 (2009).

\section{Tables}

Table 1 Partial ADME values and chemical component of the 9 post-screening components.

\begin{tabular}{llllll} 
Components & HIA & Caco-2 & HOB & PPB $100 \% \triangle$ & Chemical component \\
\hline formononetin & +0.9911 & +0.9313 & +0.5714 & 1.138 & isoflavones \\
\hline daidzein & +0.9893 & +0.9313 & +0.5714 & 0.831 & isoflavones \\
\hline Scoparone & +0.9916 & +0.8389 & +0.7143 & 0.986 & coumarins \\
\hline 3'-Methoxydaidzein & +0.9911 & +0.886 & +0.6 & 1.055 & isoflavones \\
\hline Sitosterol & +0.993 & +0.5385 & +0.5286 & 1.124 & alkaloids \\
\hline dimethylesculetin & +0.9812 & +0.5962 & +0.6143 & 1.144 & coumarins \\
\hline Coumestan & +0.9785 & +0.5843 & +0.7429 & 0.91 & coumarins \\
Capronic acid & +0.8417 & +0.8296 & +0.7429 & 0.232 & Acid
\end{tabular}

Table 2 Affinity score of the screened 5 ligand molecules to 7 receptor proteins.

\begin{tabular}{llllllll} 
Compound & \multicolumn{9}{l}{ affinity score(kcal/mol) } \\
& EGFR & JAK2 & MAPK14 & NFKB1 & ESR1 & IL2 & SRC \\
\hline Scoparone & -6 & -7 & -6.6 & -5.5 & -7.1 & -5.8 & -5.1 \\
\hline Sitosterol & -6.2 & -8.1 & -7.5 & -6.5 & -7.2 & -7.2 & -6.5 \\
\hline formononetin & -7.5 & -8.5 & -8.2 & -6.6 & -8.7 & -6.6 & -6.2 \\
\hline daidzein & -7.5 & -9.5 & -7.8 & -6.8 & -8.5 & -6.4 & -6.4 \\
\hline 3'-Methoxydaidzein & -7.6 & -8.7 & -8.9 & -6.9 & -8.8 & -6.4 & -6.4
\end{tabular}

\section{Figures}


A dry root of the Pueraria lobata (Willd.) Ohwi
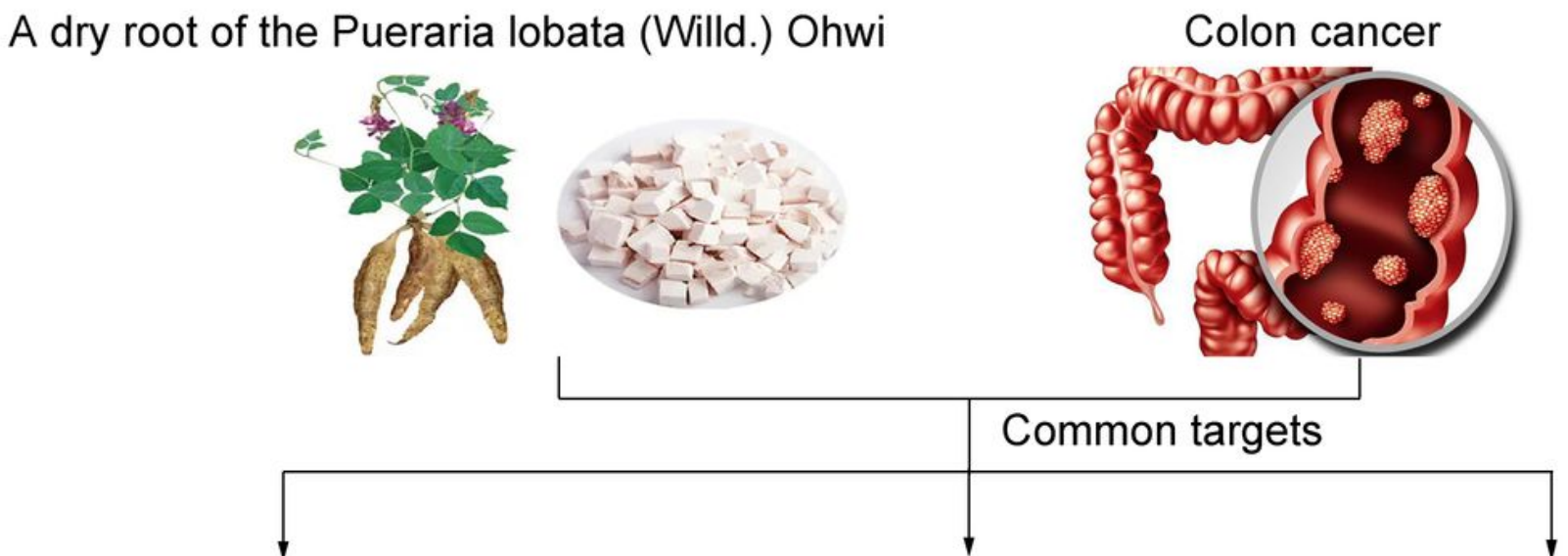

Construction of PPI network
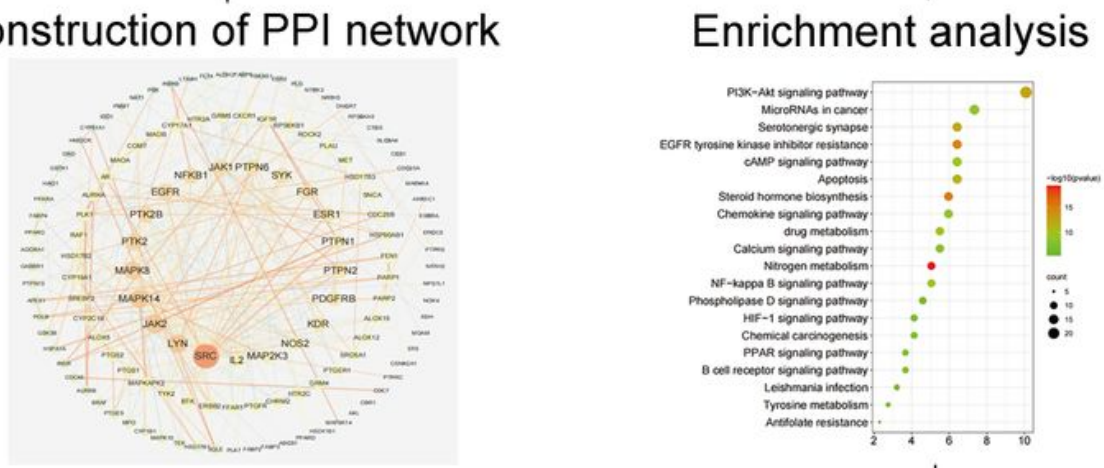

Compounds network analysis

Validation
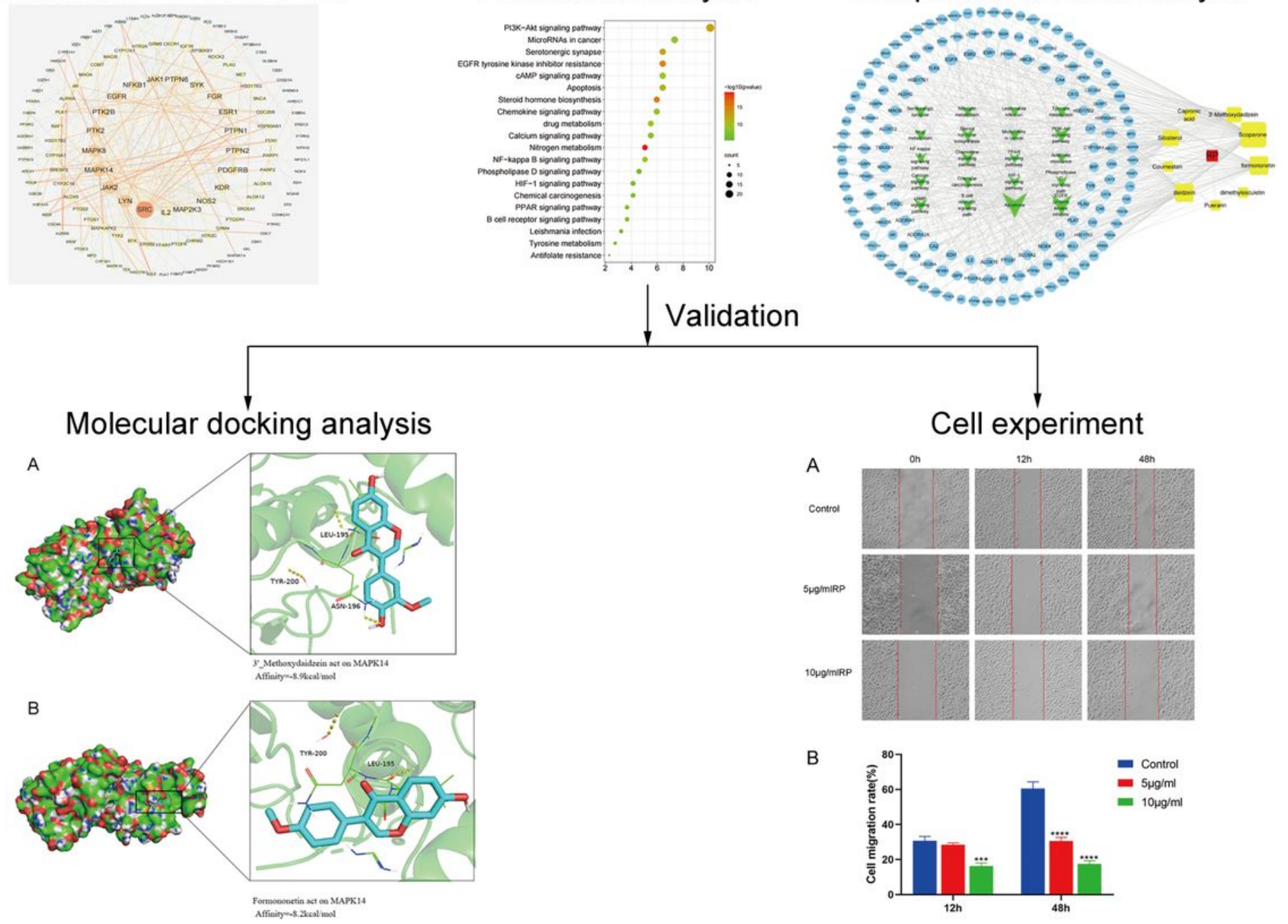

Figure 1

Flow chart for network pharmacology analysis and validation of RP anti-CC. 


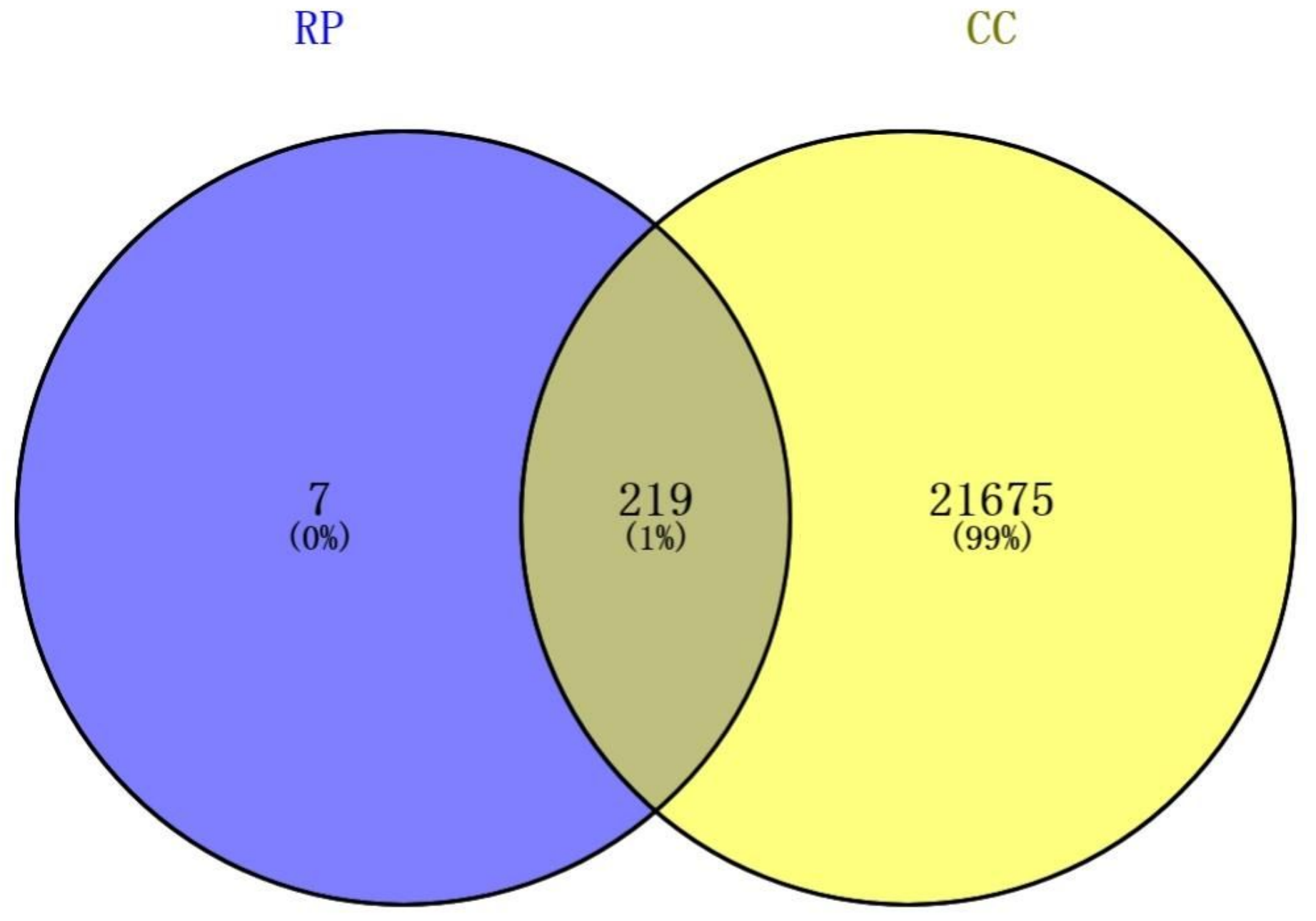

Figure 2

Venn diagram of RP and colon cancer, with 201 overlapping targets. 


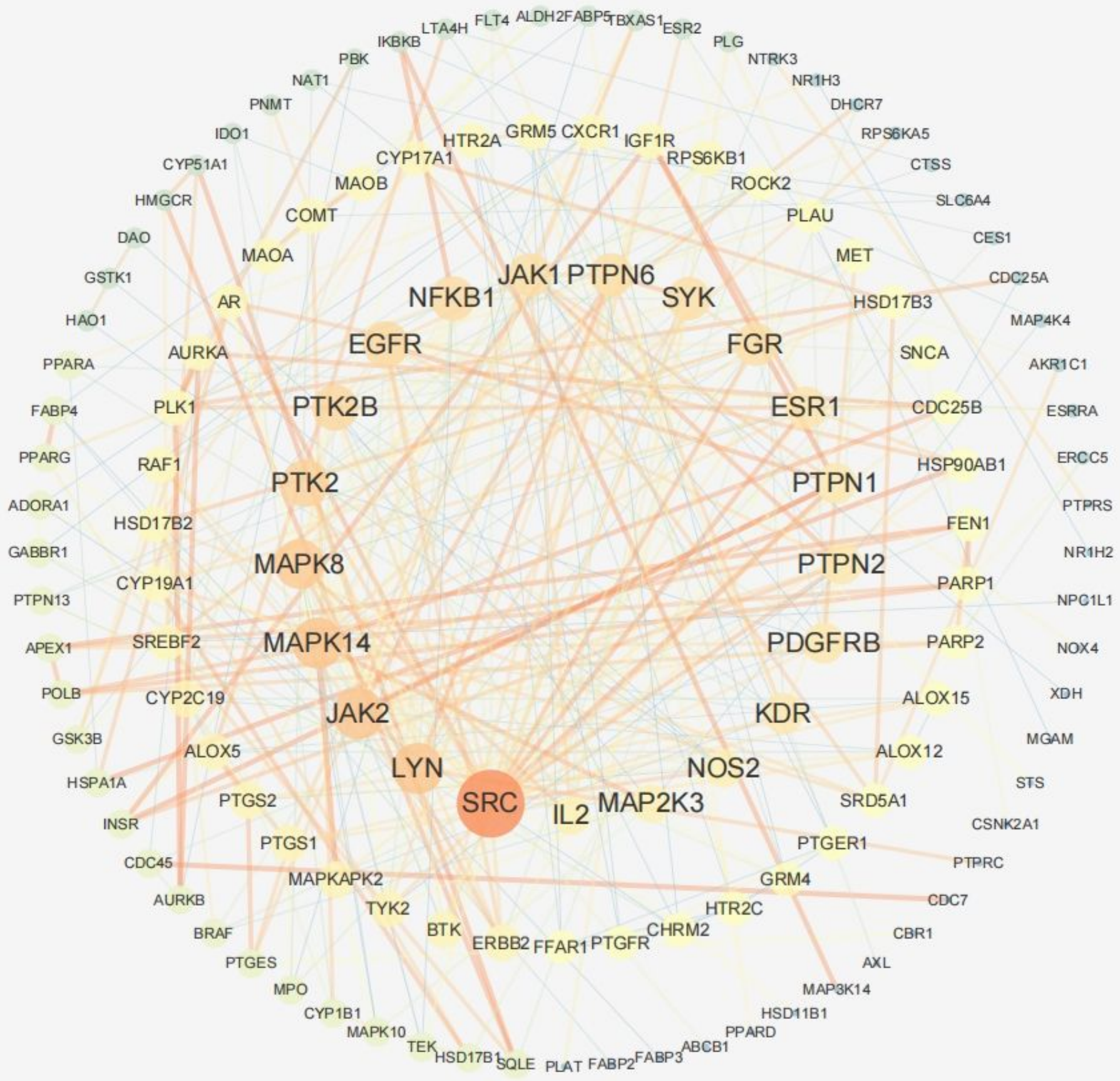

Figure 3

Visualization analysis of target PPI network. 


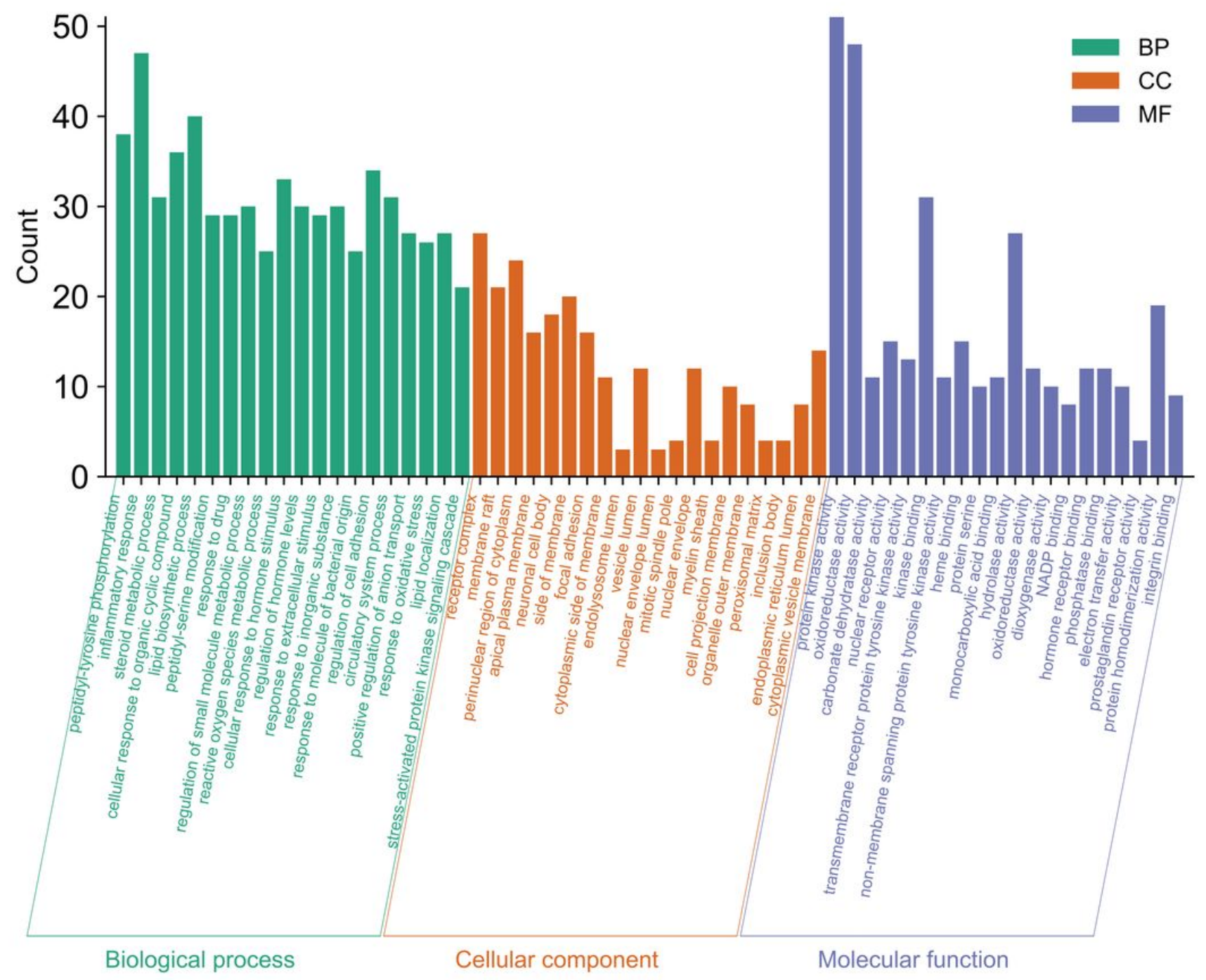

Figure 4

GO enrichment analysis of 219 targets related to RP and CC common targets. The $x$ axis represents GO terms, $y$ axis represents the number of genes enriched in each GO term $(p<0.01)$. 


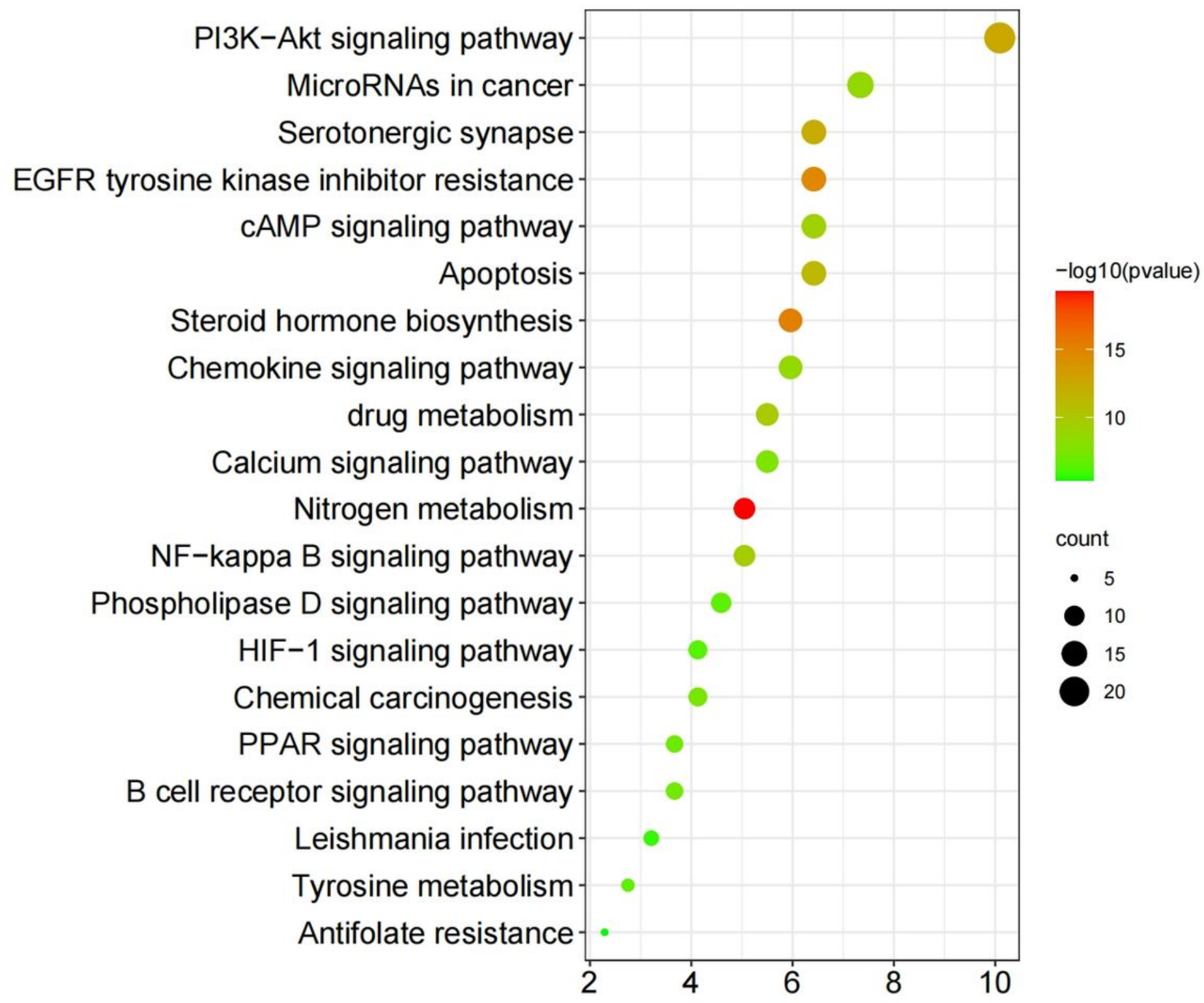

Figure 5

KEGG pathway analysis. The X-axis refers to the number of enriched targets in a pathway as a percentage of total targets, and the $\mathrm{Y}$-axis refers to the enrichment pathways. The size of the points depends on the number of targets enriched in a pathway. The color of the points depends on $\log 10(p$ value). The darker the color, the more significant the difference. 


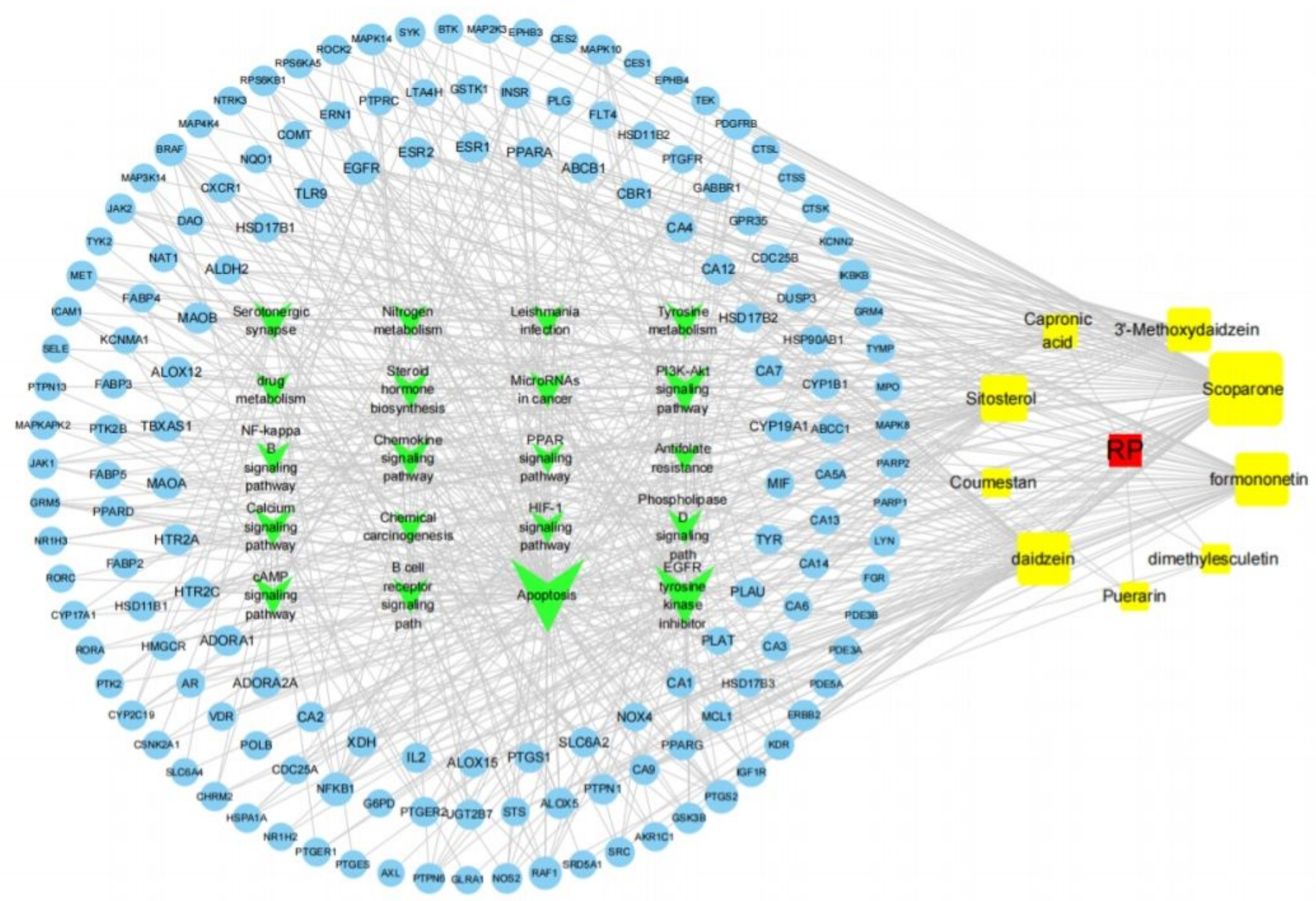

Figure 6

RP-components-targets-pathways network analysis. The red node refers to drug; The green node represents pathways; The blue nodes represent targets; The yellow nodes indicate components. The edges indicate their interactions. 


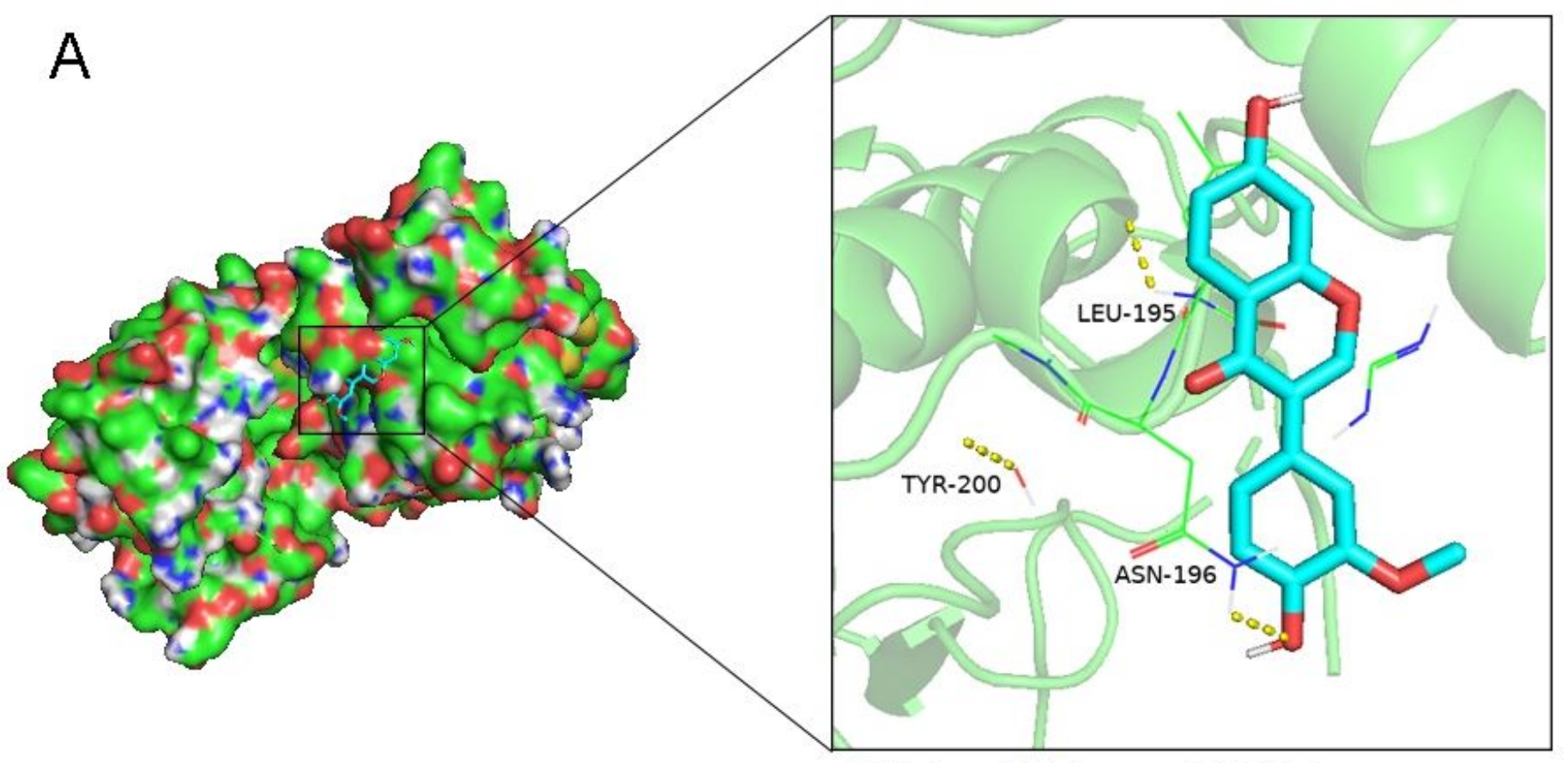

3'_Methoxydaidzein act on MAPK14

Affinity $=-8.9 \mathrm{kcal} / \mathrm{mol}$

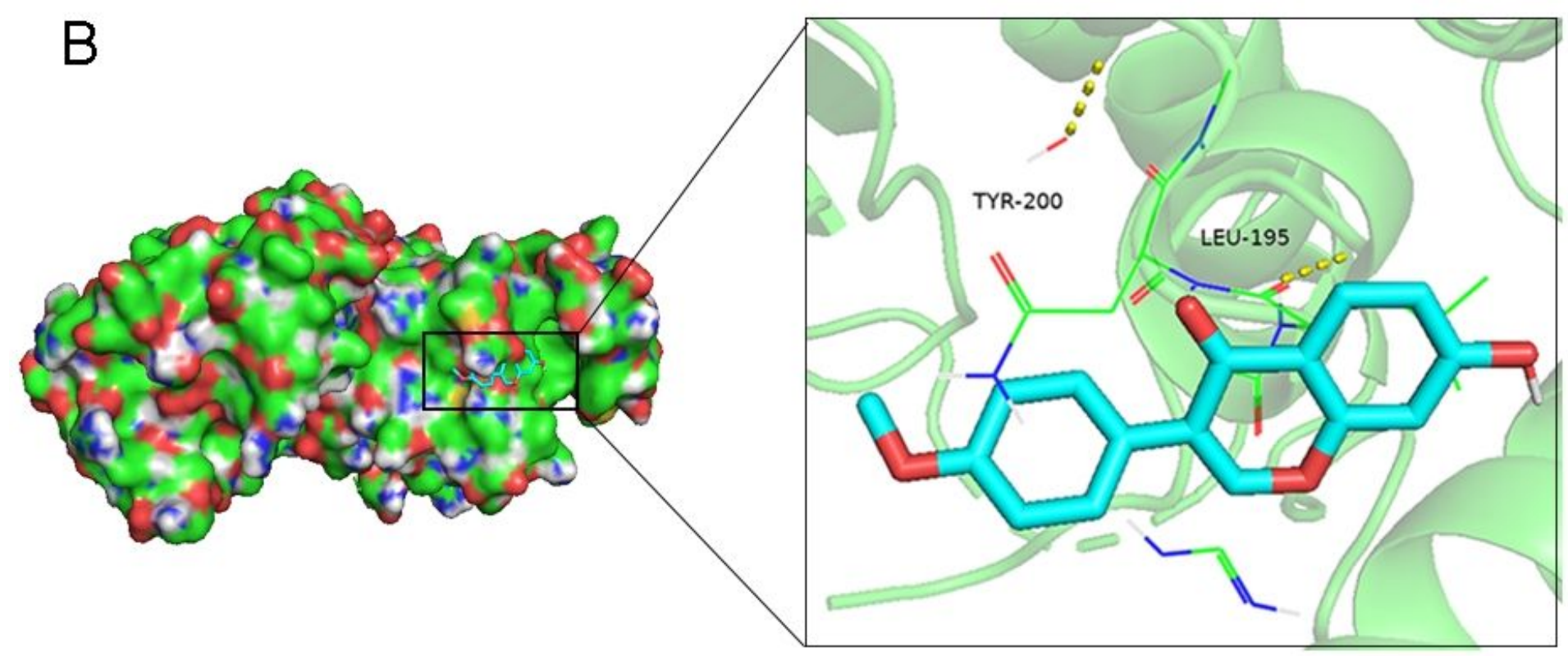

Formononetin act on MAPK14

Affinity $=-8.2 \mathrm{kcal} / \mathrm{mol}$

\section{Figure 7}

The docking complexes of ligand and receptor proteins and their binding residues are shown using PYMOL software. (A) The interaction between MAPK14 and 3'_Methoxydaidzein is through amino acid residues LEU-195, TYR-200, and ASN-196.(B) The interaction between MAPK14 and formononetin is through amino acid residues LEU-195, TYR-200. 


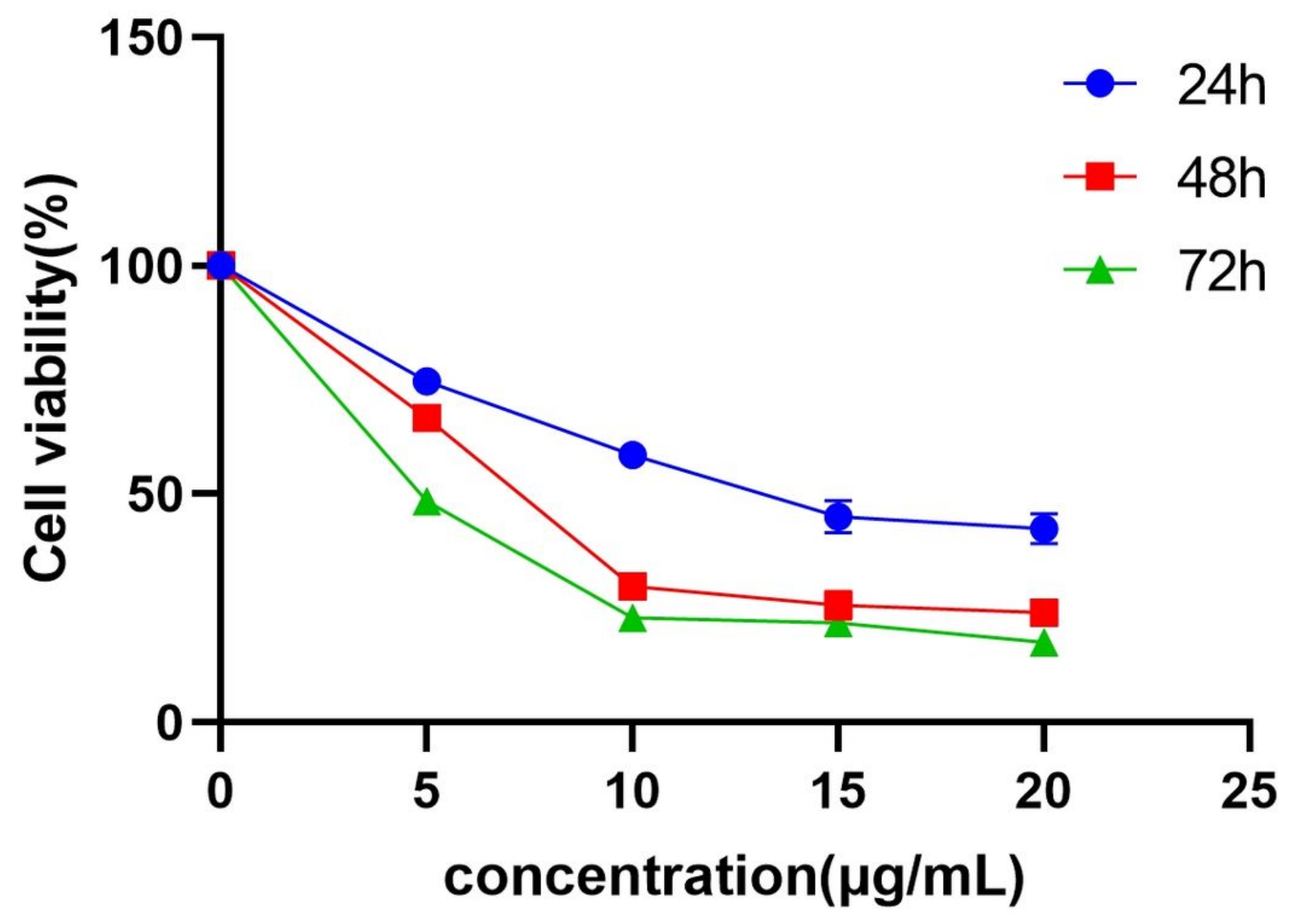

Figure 8

Through the CCK-8 experiment, RP inhibited the proliferation of CC cells. SW480 cells were treated with $\mathrm{RP}$ at different concentrations for 24,48 \and 72 hours respectively. The data is expressed as the mean \pm SD of three separate experiments. 
A

Oh

$12 \mathrm{~h}$

$48 \mathrm{~h}$

Control
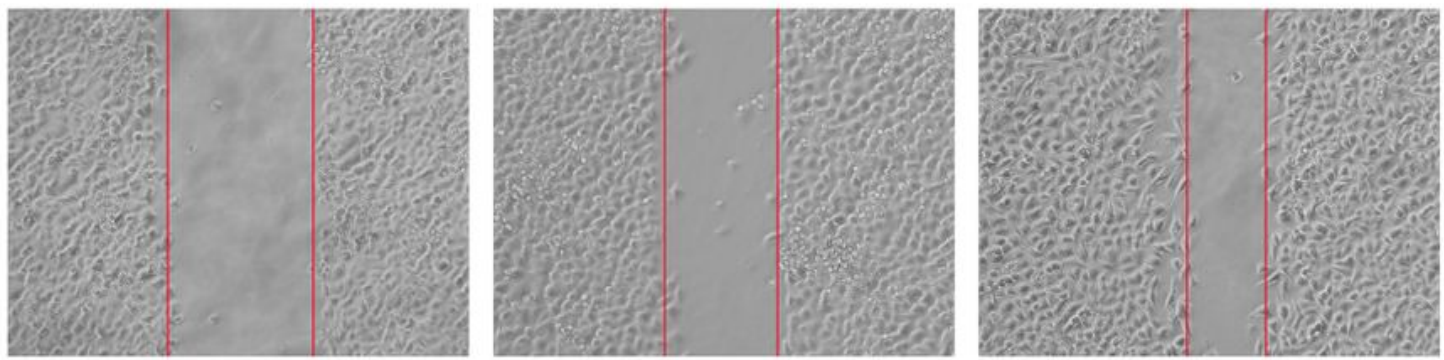

$5 \mu \mathrm{g} / \mathrm{mIRP}$
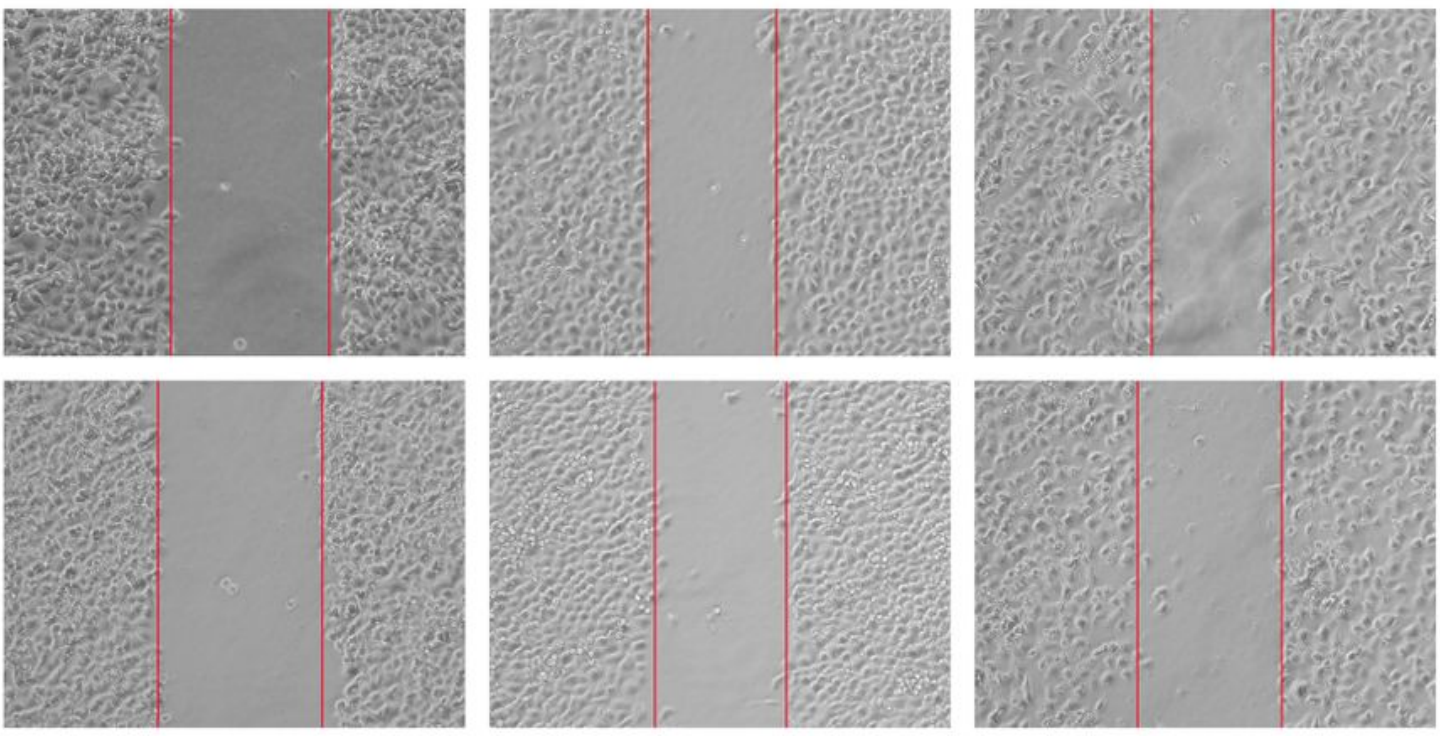

$10 \mu \mathrm{g} / \mathrm{mIRP}$

B

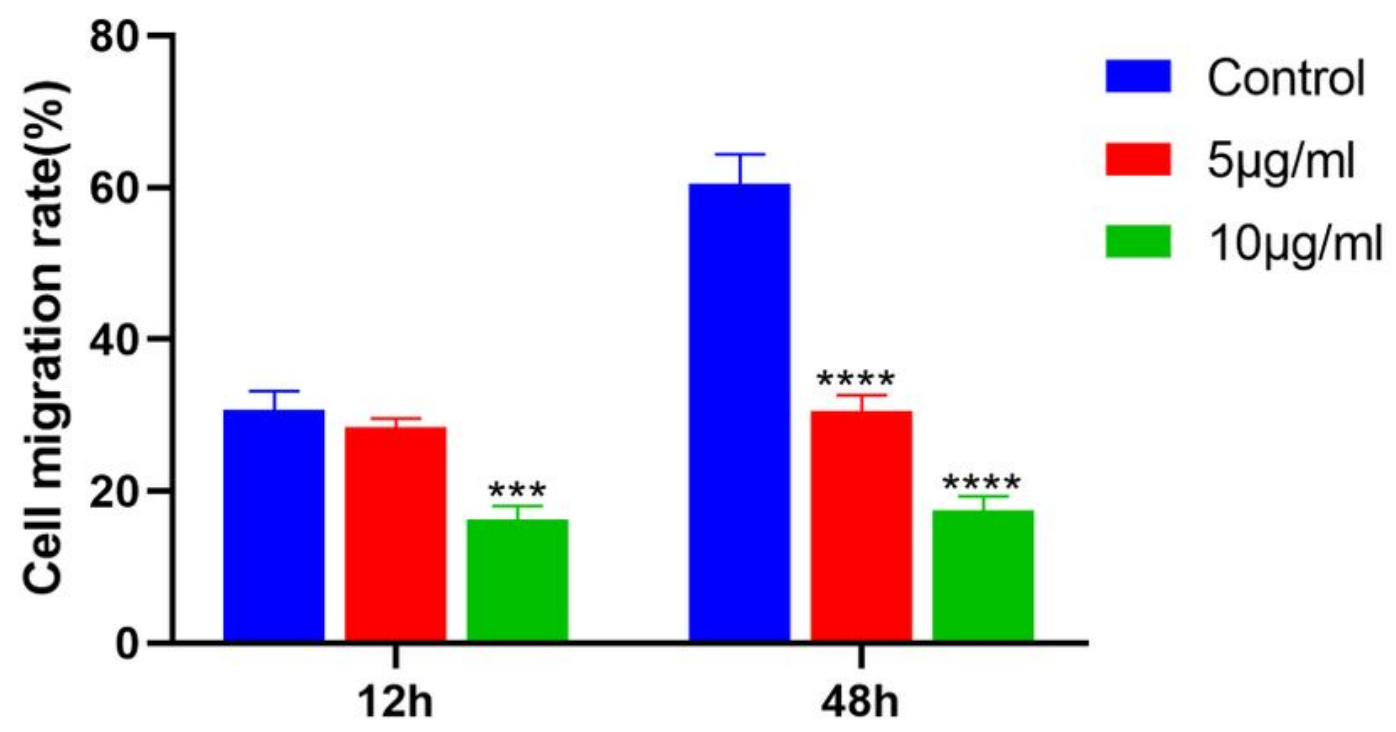

Figure 9

RP significantly inhibited the migration of SW480 cells. The effect of RP $(0,5,10 \mu \mathrm{g} / \mathrm{ml})$ on SW480 cell migration activity was examined under an inverted microscope to evaluate cell migration (40x). (A) Images during cell migration in scratch experiments. (B) The average scratch cell mobility at $12 \mathrm{~h}$ and 48 $\mathrm{h}$ after $5 \mu \mathrm{g} / \mathrm{ml}$ and $10 \mu \mathrm{g} / \mathrm{ml}$ treatment. ${ }^{\star \star \star} \mathrm{P}<0.001$, ${ }^{\star \star \star \star} \mathrm{p}<0.0001$ compared with control $(0 \mu \mathrm{g} / \mathrm{ml})$. 


\section{Supplementary Files}

This is a list of supplementary files associated with this preprint. Click to download.

- SupplementaryTables.pdf 